\title{
Sistema para las tutorías académicas en las universidades ecuatorianas. Caso Universidad Estatal de Milagro
}

\section{System for academic tutorials in Ecuadorian universities. Case State University of Milagro}

Mirella Azucena Correa-Peralta

Jorge Luis Vinueza_Martínez

Erika Maribel Torres Arias

Karina Elizabeth Ponce Intriago

Universidad Estatal de Milagro, Ecuador

Autor por Correspondencia: mcorreap@unemi.edu.ec,jvinuezam@unemi.edu.ec, etorresa@unemi.edu.ec,kponcei@unemi.edu.ec

Fecha de recepción: 30 de Marzo de 2017 - Fecha de aceptación: 5 de Junio de 2017

Resumen: El desarrollo de esta investigación parte de la necesidad de incluir a docentes universitarios en las actividades de tutorías académicas, actividad que lo estipula el Reglamento de Escalafón Docente en el Ecuador. El objetivo es identificar la incidencia de los procesos de tutorías académicas en las universidades para ser sistematizadas. Este estudio, se efectuó en la Universidad Estatal de Milagro en el 2014, aplicando encuesta a 1256 estudiantes, 126 docentes y 28 directivos, analizándose: 1. Procesos, 2. Estandarización y 3. Aplicación de las tutorías académicas. El 97\% de los estudiantes coinciden que debe existir el acompañamiento académico con docentes especializados $(70 \%)$ y con una comunicación fluida (90\%). Como producto se presenta un diagrama de proceso y un diagrama en UML, concluyéndose que la tecnología aporta en la asignación de horarios, seguimiento y comunicación de las tutorías académicas presenciales y virtuales.

Palabras clave: tutorías académicas; docentes-tutores; estandarización

Abstract: The development of this research starts from the need to include university teachers in academic tutoring activities, an activity that is stipulated by the Regulation of Teaching Staff in Ecuador. The objective is to identify the incidence of academic tutoring processes in universities for to be systematized. This study was carried out at the State University of Miracle in 2014, applying a survey to 1256 students, 126 teachers and 28 managers, analyzing: 1. Processes, 2. Standardization and 3. Application of academic tutorials. $97 \%$ of the students agree that there must be academic accompaniment with specialized teachers (70\%) and fluent communication (90\%). As a product, a process diagram and a UML diagram are presented, concluding that the technology contributes in the assignment of schedules, monitoring and communication of academic and virtual tutorials.

Key words: academic tutorials; teachers-tutors; standardization 


\section{Introducción}

Las Instituciones de Educación Superior están enfocadas en elevar el nivel de eficiencia en los servicios, ya sea por el avance de las tecnologías de la información, la acreditación universitaria o las exigencias del medio. En el caso del Ecuador, en el Reglamento de Carrera y Escalafón del profesor e investigador del Sistema de Educación Superior (2012), en su Artículo 6, establece las actividades del docente, entre las cuales menciona dar "Orientación y acompañamiento a través de tutorías presenciales o virtuales, individuales o grupales"; es decir que existan docentes guías-tutores para acompañar a los estudiantes en actividades académicas. El propósito de esta investigación es que los docentes tutores tengan un sistema de apoyo como guía en temas académicos ya sea dentro o fuera del aula desde un balance de participación activa en la vida universitaria.

Desde el enfoque constructivista y con aprendizaje significativo subyace el conocimiento, por ello la importancia de mejorar la relación docente-estudiante a nivel superior como el aprendizaje cooperativo, por citar el método de CO-OP CO-OP (Kagan 1985) desde la coevaluación grupal e individual y evaluación del producto.

Cabe mencionar, que se espera disminuir el fracaso escolar, deserción o baja productividad, que cobra de gran importancia a la calidad educativa para el desarrollo cognitivo desde la metodología de interacción, organización del trabajo y estrategia de socialización y sus valores (Lara V, 2005). Este esfuerzo es constante para cumplir con las actividades académicas, pues en ocasiones debido a problemas externos, a lo académico, los estudiantes se desmotivan y pierden el interés por cumplir con las actividades asignadas, con la probabilidad de que existan momentos críticos que alteren su desempeño.

Para Rodríguez (2003), el sistema de tutores inició en el siglo XVI desde la Universidad Oxford, basado en la relación estrecha tutor-estudiante; además la historia ha representado personalidades que usaron tutorías como Confusio, Platón, Sigmeund Freud, entre otros. En este sentido la universidad tiene como reto alcanzar excelencia académica, que implica no solo en instruir, sino formar en educación para explotar el proceso de enseñanza-aprendizaje (Blimling, 2000). Como lo menciona Rey C., Balagué, N. y Falomir, V. (2006) las universidades han de saber evolucionar como entidad de investigación y desde la cultura científica y humanística; es decir, una evolución que aborde la evidencia de resultados y logros orientado a los usuarios Balagué, N. y Saarti, J. (2009),

Pérez R (2012), menciona que el contar con un sistema de tutoría universitaria puede ser un instrumento efectivo para la construcción tutelada del aprendizaje de forma autónoma, con recursos disponibles que cumplan las necesidades independientes del estudiante, esta premisa tiene coincidencia en lo expuesto por Domínguez, Álvarez y López, (2013); Lantarón (2014); Pérez Cusó, J, Martínez Juárez. M, (2015).

Esta investigación promueve el conocer: ¿De qué forma las actividades de docentes tutores son satisfactorios?, de ahí los objetivos: analizar las causas que inciden en los procesos de las tutorías académicas universitarias. 
Qué han hecho las universidades para aplicar la normativa que regula a las instituciones de educación superior, necesariamente como respuesta es incluir estrategias; para ello la Agencia Nacional de Evaluación de la calidad y Acreditación (ANECA) publicó el Manual de la evaluación docente (2009) donde incluye el rol del docente-tutor desde la planificación, metodología, gestión de la docencia, valoración general de la tutoría, sistema de evaluacion de asignatura, valoración de la actividad del equipo docente, todo con la finalidad no solo ejecutar una actividad, sino evaluar resultados desde la orientación de asistencia académica.

La Universidad de Veracruz, México (2012), se encuentran archivos acerca del sistema tutorial relacionada con el seguimiento de la trayectoria escolar del alumno, para orientarlo en la toma de decisiones y la enseñanza como apoyo estudiantil, desde el contacto docenteestudiantes.

La Asociación Nacional de Universidades e Instituciones de Educación Superior (ANUIES) (Cruz G, Chehaybay E. Abreu F. (2011)), invoca las deficiencias de la actividad de tutoría por la falta de documentos en el proceso tutorial y la interacción entre tutor-tutorado (Eliasson, Berggren y Bondestam, 2000). Desde el enfoque de ANUIES refiere además, la falta de claridad de metas y objetivos en la educación superior para clarificar procesos eficaces. No obstante el anclaje estructural, pragmático y logístico genera la organización de las tutorías universitarias (Figura 1).

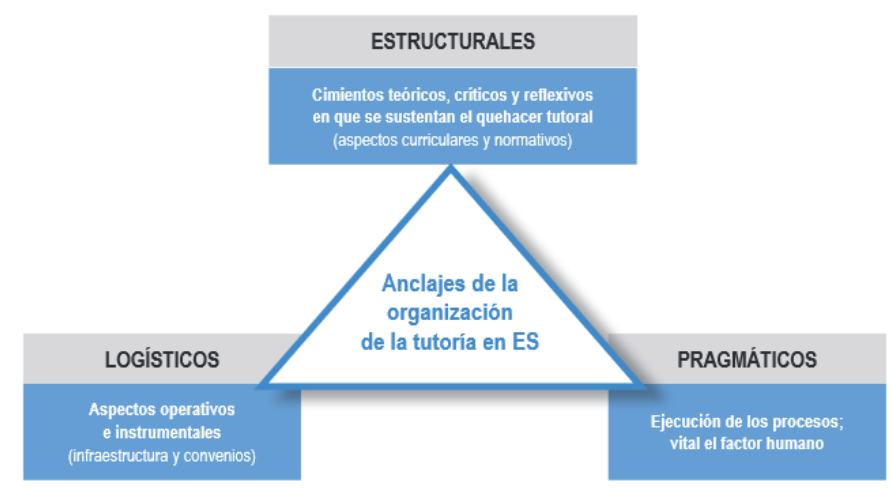

Figura 1: Anclaje de la organización de la tutoría en educación superior

Fuente: Tomado de Tutoría en educación Superior: Una revisión analítica de la literatura de Cruz G, Chehaybay E., Abreu F. (2011)

En la Constitución del Ecuador (2013) menciona a la educación como un derecho y prioritario en la política pública y la inversión estatal, con una participación del proceso educativo de las personas, las familias y la sociedad tienen el derecho y la responsabilidad de participar en el proceso educativo.

En el Ecuador, los docentes tutores de aula de Bachillerato, según el Reglamento General a la Ley Orgánica de Educación Intercultural (2012) artículo 56, indican que los tutores ayudan a evaluar el desempeño estudiantil y a quienes ingresarán a los estudios universitarios, además coordinar actividades académicas, deportivas, sociales y culturales.

Además, la universidad debe aumentar la pertinencia desde la evaluación formativa ya sea con proyectos institucionales, gestión académica efectiva, para la implementación de 
sistemas tecnológicos en un escenario fundamental para las instituciones de educación superior (Molina L; Rey C; Vall A; Clery A; Santa María G; 2016).

\section{Metodología}

Hayes, S. y Ljungberg, M. (2011) realizaron un estudio del estado cualitativo en la construcción, transformación y comprensión en relación al tiempo y recursos necesarios; es ahí que este estudio es un referente desde el espacio de reflexión de Tutorías Académicas, que fue el referente para este estudio.

El trabajo de investigación, se efectuó en la Universidad Estatal de Milagro en el 2014, con un diseño casi experimental descriptivo, tipo encuesta dirigido a estudiantes, docentes y directivos, distribuidos por Facultades Académicas. Se desarrolló en un primer momento de forma cualitativa identificando los procesos, estandarización y la aplicación de las tutorías académicas.

El proceso fue por fases: 1) Revisión de fuentes en relación al ámbito de estudio, 2) Definición de los objetivos, 3) Determinación de la población e instrumento a aplicar, 4) Diseño y validación del instrumento (cuestionario), 5) Recoger información (estudiante, docentes y directivos), 6) Análisis de los resultados, y 7) Comunicar los resultados.

La claridad y consistencia de la encuesta fue evaluada por 3 personas; y de ahí se determinó la muestra, aplicando la fórmula de distribución, con un nivel de confianza del 95\%, un error del $5 \%$, probabilidad p y q de 0,5 a una población de 4081, obteniendo una muestra de 1256 (ver Tabla 1). La recolección y procesamiento de la información se utilizó un formulario para facilitar la tabulación.

$$
N=\frac{n p q}{\frac{(n-1) E^{2}}{Z^{2}}+p q}
$$

n: tamaño de muestra.

$\mathrm{N}$ : tamaño de población

$\mathrm{p}$ : posibilidad de que ocurra un evento, $\mathrm{p}=0,5$

q: posibilidad de no ocurrencia de un evento, $q=0,5$

E: error se considera el $5 \%$ : $\mathrm{E}=0,05$

$\mathrm{Z}$ : nivel de confianza, que para el $95 \%, \mathrm{z}=1,96$

Tabla 1: Número de estudiantes por Facultades en la Universidad Estatal de Milagro.

\begin{tabular}{lrr}
\hline Facultad & $\boldsymbol{N}^{\circ}$ de Estudiantes & Distribución de la muestra \\
\hline Ciencias de la Salud & 922 & 271 \\
Ciencias Administrativas & 1275 & 295 \\
Ciencias de la Educación & 952 & 273 \\
Ciencias de la Ingeniería & 561 & 228 \\
Semipresencial y a distancia & 371 & 189 \\
TOTAL & 4081 & 1256 \\
\hline
\end{tabular}

Fuente: Secretaria General, Septiembre 2014. 


\section{Resultados}

En la Universidad Estatal de Milagro, la encuesta se aplicó a 1256 estudiantes, 28 directivos y 126 docentes, al ser objetos de la investigación Hammersley, M. y Atkinson, P. (2009). El análisis de datos cuantitativos se efectuó en los aspectos de: procesos, estandarización y aplicación de las tutorías académicas.

\section{Resultados a los procesos}

El 97\% de los estudiantes están de acuerdo con el acompañamiento académico, 54\% conocen de las tutorías académicas, $30 \%$ recibió tutorías; sin embargo el 5\% fueron informados de los horarios; de ahí, que el 96\% consideran necesario apoyarse con un sistema para la gestión de tutorías.

En el caso de los docentes el $87 \%$ desconocen las políticas, formatos o reglamentos, $26 \%$ conocen el proceso y las actividades de un docente tutor; también el $99 \%$ están totalmente de acuerdo contar con el proceso, sistematización y repositorio para la gestión de las tutorías.

Para los directivos el $68 \%$ desconoce que existan políticas o formatos y están de acuerdo con un sistema que ayude al proceso y socialización del mismo.

En relación a mejorar el proceso de la gestión de tutorías, se observa en la Figura 2 incluyendo un desviación estándar tiende a $0 \%$, el proceso de conocimiento de tutorías académicas, (estudiantes, docentes y directivos) del proceso de tutorías es bajo, pues en promedio general $33 \%$ desconocen y $59 \%$ algo.

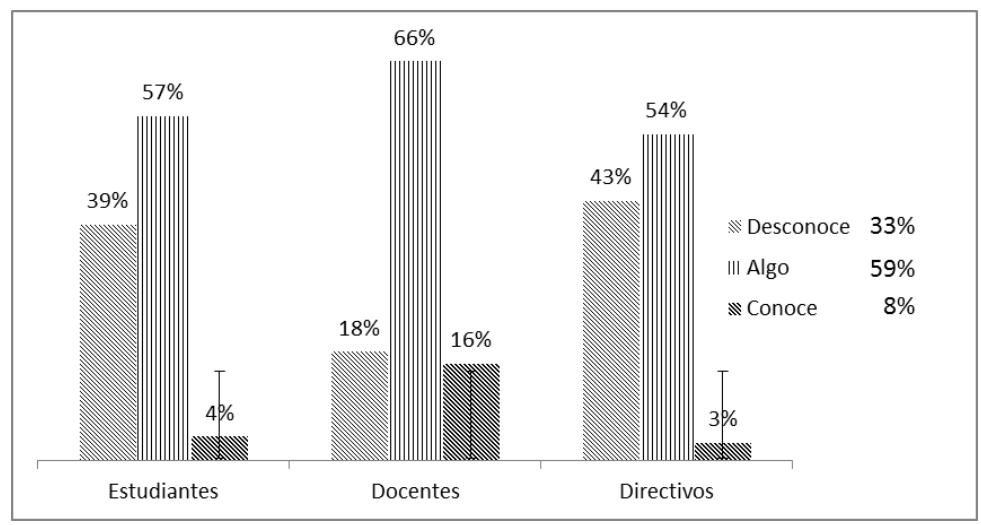

Figura 2: Conocimiento del Proceso de las tutorías académicas en la Universidad Estatal de Milagro Fuente: Encuesta del proceso de asignación de docentes tutores, 2014

\section{Resultado de la estandarización.}

En cuanto a la estandarización, el $62 \%$ de los docentes y directivos académicos tienen identificado que las políticas, formatos o reglamentos son parte de la estandarización del 
proceso; e inclusive el conocimiento, ejecución y comunicación impactan en un $92 \%$ al proceso académico (Figura 2 y Figura 3).

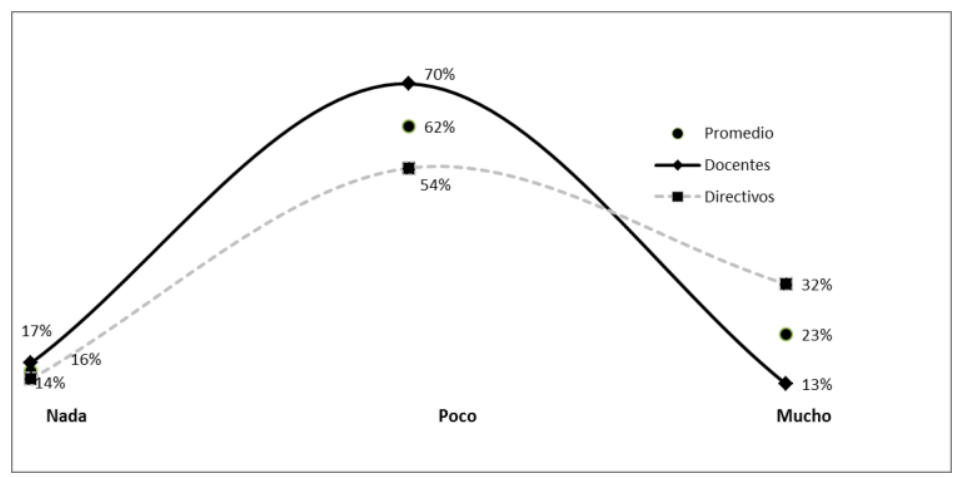

Figura 3: Conocimiento de políticas, formatos o reglamentos para estandarizar el proceso de tutorías académicas Fuente: Encuesta del proceso de asignación de docentes tutores, 2014

De acuerdo a los datos obtenidos y comparando las variables de procesos, políticas y actividades, se presentó que existe influencia en un 50\% (con escala de Likert -nada-0, poco 0,5 y Mucho 1), generando una alta incidencia de las carreras no presenciales con el 56\% (Figura 4).

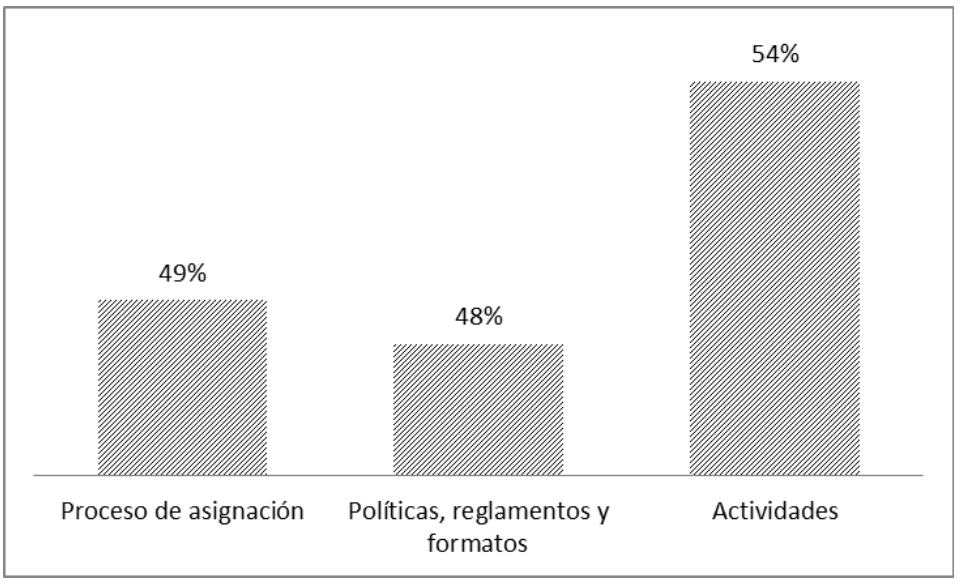

Figura 4: Conocimiento de los Procesos, políticas y actividades para la estandarización del proceso de tutorías académicas.

Fuente: Encuesta del proceso de asignación de docentes tutores, 2014

\section{Desde la aplicación}

El 70\% de los estudiantes consideran que los docentes especializados deben dar asesoramiento académico, el $90 \%$ cataloga que la comunicación debe ser fluida para la gestión de los docentes tutores; es así que los estudiantes, docentes y directivos están de acuerdo con un sistema para la gestión de tutorías académicas en la universidad.

La propuesta aborda la representación en el contexto universitario desde la gestión del conocimiento, ya sea en transportar o adquirir información o conocimiento (Pérez-Montoro M., 2004). Es así que para las universidades es importante identificar mejoras desde su alcance académico, para este fin formula en contar con un escenario tecnológico relacionado a mejorar el acceso de los estudiantes, mejorar la relación académica con gestión académica eficiente, con 
sustento a normativas legales. Como lo menciona Díaz J, Torres W, Orozco W (2016) incorporar la gestión académica acorde al conectivismo, neuroaprendizaje y pedagogía crítica con formación holística, participativa y creativa.

Como se observa en la Figura 5, se planteó el orden de las actividades e inclusión del sistema de gestión de tutorías académicas en una organización de planificar, ejecutar, verificar y actuar para tomar las mejores experiencias que involucre resultados beneficiosos.

Posteriormente, se analizó mediante el diagrama de modelado de sistemas de software denominado Lenguaje Unificado de Modelamiento (UML) para incluir aspecto general de sistematización de los objetos (Ver Figura 6).

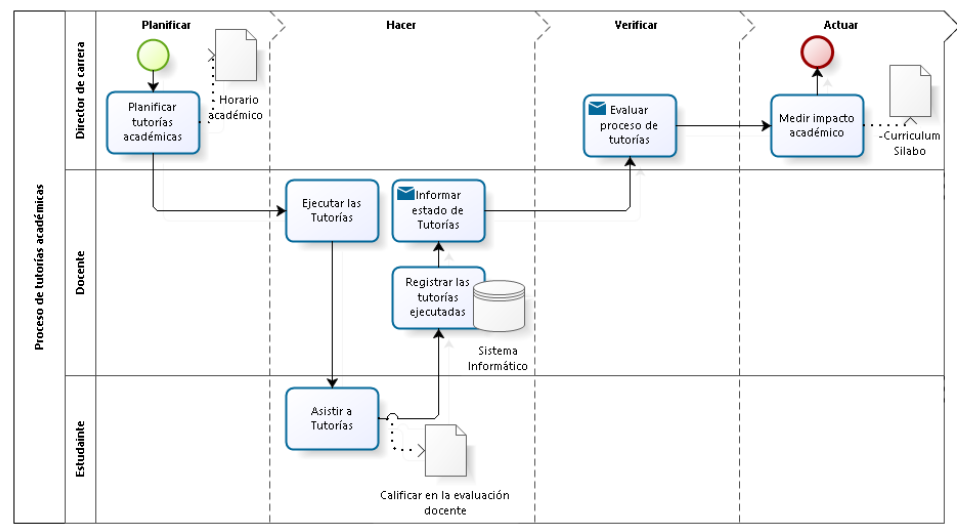

Figura 5: Proceso de tutorías académicas - UNEMI.

Fuente: Adaptado proyecto de grado Ponce K, Torres E (2014). Diseño de un Sistema Web para la automatización de los procesos en la asignación de docentes tutores de aula en la Universidad Estatal de Milagro periodo 2014.

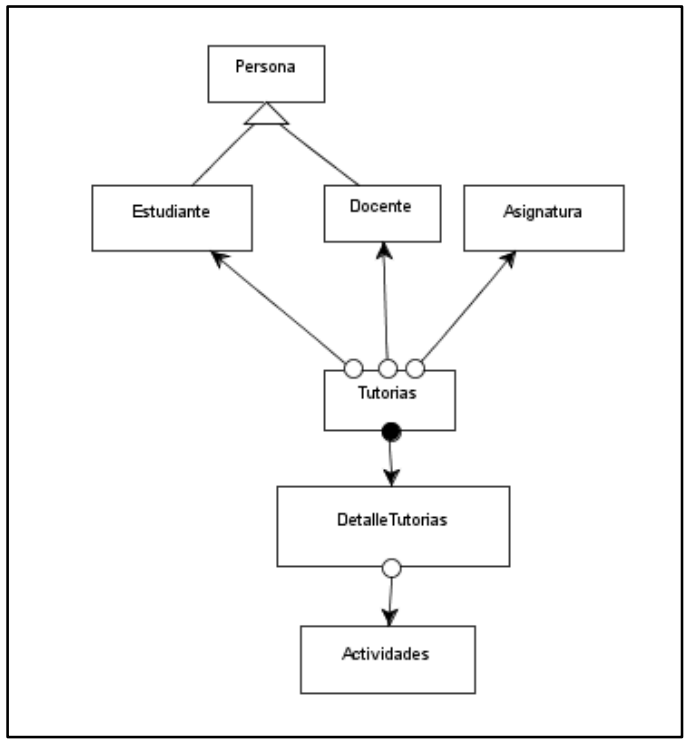

Figura 6: Diagrama de objetos UML de la sistematización de tutorías académica

\section{Discusión}


El desarrollo de la sociedad de la información, de la economía del conocimiento e internacionalización de la educación universitaria, han cuestionado el abordaje de las tutorías en las universidades (Villena D., Muñoz A y Polo M., 2013), desde el ámbito del Reglamento de Régimen Académico en las actividades de aprendizaje asistido por el profesor está el desarrollar actividades en las modalidades en línea y a distancia, con una tutoría sincrónica; en el caso del aprendizaje colaborativo incluye las tutorías en procesos colectivos para el aprendizaje con el uso de la tecnología, metodologías en red, tutorías en situ o en entornos virtuales (CES, 2013).

En el Reglamento de Carrera y escalafón del profesor e investigador del Sistema de Educación Superior codifica en 2016), los docentes universitarios tendrán dentro de sus actividades la "Orientación y acompañamiento a través de tutorías presenciales o virtuales, individuales o grupales" (CES, 2012).

Ante los retos de la era digital la función del docente no solo es de transmitir sino de ayudar para aprender en la construcción del conocimiento. El apoyo cercano del docente tutor crea un nivel de confianza generando nuevas competencias profesionales en el rol del docente (Pérez, 2012).

En este trabajo de investigación, los participantes coinciden de la necesidad de generar el proceso y sistematizar como apoyo a las tutorías académicas en la universidad, evitando la improvisación. En el caso de otros estudios en relación a tutorías universitarias en Reino Unido los estudiantes asisten a tutorías como recurso importante ante el apoyo académico (Cashmore, A., Scott, J., y Cane, C., 2012), que es considerado en este estudio.

El rol de las autoridades es preciso, en aras de establecer modelos de comunicación que contribuya a los procesos académicos, generando un desarrollo de modelos para mejorar desde la planificación a la ejecución de los resultados, involucrando un espacio cercano del estudiante para atención individualizada y grupal en aspectos académicos y promover la conexión simultánea (Villena D., Muñoz A y Polo M., 2013).

El estudio de Lantarón (2014) menciona el estudio referente a las tutorías individuales o grupales con un mínimo de $2,2 \%$ de servicios que indica no mantener contacto con los tutores, que no coincide con el presente estudio pues las tutorías no la reciben en un 24\%; por lo que se coincide con Sánchez y Guillamón (2008) referente al impacto de las tecnologías en los procesos de tutorías académicas.

El trabajo de investigación se centra en conocer la opinión de los estudiantes y docentes del servicio de tutoría universitaria, no se ha tratado de la satisfacción posterior con la aplicación y mejora del sistema, identificar la opinión de los estudiantes sería objeto de otro estudio.

En la acción tutorial, las universidades deben ser consiente en las dimensiones de aprendizaje, apoyo, autonomía, comunicación, aula estimulante, grupos de estudios, metodología adecuada, coordinación académica y los espacios en relación al currículum; es decir promover un espacio universitario desde la transición de bachillerato a universidad para contribuir a la transformación social (Calvo A; Rodríguez C; Haya I; 2013). 
De esta manera los resultados obtenidos presentan un escaso conocimiento de procesos, políticas y actividades por parte de los docentes y estudiantes; estos datos están en proporción con los estudios de Sanz Díaz (2012) como es el tiempo de dedicación del alumno, cercanía del profesor, tiempo de dedicación, y principalmente la importancia de las tutorías (43\%), y principalmente el desconocimiento de los servicios de tutorías o no haber utilizado el servicio.

\section{Conclusión}

A manera de conclusión, se coincide con el estudio de Sanz Díaz (2012), que los estudiantes consideran asistir a las tutorías para aclarar dudas académicas, cercanía con el docente, facilitar el estudio, atender orientación o motivación académica, utilizando la asistencia in situ. Además concuerdan que no asistían a las tutorías por afectación de los horarios, por lo que sugieren flexibilidad, siendo similares consideraciones como asistencia a las tutorías, publicación de horarios, organización de las tutorías, entre otras.

Con el uso de las tecnologías aportaría a la asignación de horarios, continuidad e incentivo de la comunicación con aspectos de tutorías presenciales y virtuales.

Las universidades están orientando sus actividades en función del avance del internet, con tutores competentes involucrando la comunicación, manejo de conflictos, trabajo en equipo y planificación del tiempo (Castaño, Blanco y Asensio, 2012). Así como el avance de experiencias y aportaciones de los estudiantes que motive a aprender para obtener un tutor con personalidad o perfil humano con preparación y dominio con técnicas adecuadas (García, E., Conejero, J. A., \& Díez, J. L., 2014).

Los estudiantes bajan el rendimiento académico por lo que sugieren acompañamientos para alternativas de mejoras, así como un esquema que incluya la atención de las diferencias basado en el interés y evaluación de competencias individuales (Bricio K, 2014; Candell J, 2015).

Los resultados sugieren que la investigación que se debe identificar las brechas para futuras investigaciones, de las brechas encontradas como aspectos de calidad en la cobertura académica adecuada, para mejorar la parte tradicional de la educación; sin embargo, incluir la tecnología que estructure y organice entornos informáticos de localización de información (Pérez-Montoro, 2010), y mantener así la comunicación colaborativa entre docente-alumno.

\section{Bibliografía}

Aneca (2009). Manual de Evaluación Docente de la Universidad Nacional de Educación a Distancia. Obtenido de http://portal.uned.es/pls/portal/docs/Page/Uned_Main/Launiversidad/Vicerrectorados/Cal idad_E_Internacionalizacion/Calidad/Evaluacion\%20actividad\%20docente/Manual_Doce ntia_Uned_Aprobado_Aneca_09_04_20.Pdf

Asamblea Constituyente. (2008). Constitución del Ecuador. Obtenido de http://www.asambleanacional.gov.ec/documentos/constitucion_de_bolsillo.pdf 
Balagué, N. y Saarti, J. (2009). Benchmarking quality systems in two European academic libraries. Library Management, 30 (4/5), p. 227 - 239

Blimling, G. S. (2000). Accountability for student affairs: Trends for the $21^{\text {st }}$ century. En C. Johnson \& H. Cheatham (Eds.), Higher Education trends for the next century: A research agenda for student success (pp. 51--56). Washington, D.C.: American College Personnel Association.

Bricio K. (2014). Incidencia del estilo de vida en el rendimiento académico de los estudiantes, mediante un análisis interno en la Universidad Estatal Península de Santa Elena UPSE, de la provincia de Santa Elena año 2014. Revista Científica y Tecnológica UPSE, 2 (2).

Candell J (2015). La profesionalización de los docentes en los procesos de acreditación de la Universidad Estatal península Santa Elena. Propuesta centro de capacitación docentemetodológico de la UPSE. 2(3)

Calvo Salvador, A; Rodríguez - Hoyos, C; Haya Salmón, I; (2013). La dimension personal de la tutoria universitaria. Una investigación cualitativa en la Universidad de Cantabria (España). Revista Española de Orientación y Psicopedagogía, 24() 98-113. Obtenido de http://www.redalyc.org/articulo.oa?id=338230795007

Cashmore A., Scott, J. \& Cane, C. (2012). "Belonging" and "intimacy" factors in the retention of students - an investigation into the student perceptions of effective practice and how that practice can be replicated. Leicester, Inglaterra: Universidad de Leicester.

Castaño, E. Blanco, A. Y Asensio, E. (2012). Competencias para la tutoría: experiencia de formación con profesores universitarios. REDU - Revista de Docencia Universitaria, 10 (2), 193-210.

Cruz G, Chehaybay E., Abreu F. (2011). Tutoría en Educación Superior: Una revisión analítica de la literatura. Revista de la Educación Superior Vol XL(1), No. 157, 189-2009

Díaz J, Torres W, Orozco W (2016). Nuevos horizontes académicos para los estudios de tecnologías de la información y las comunicaciones en Ecuador. Revista Ciencias Pedagógicas e Innovación UPSE. Volumen IV N. 1. 61-78

Domínguez, G., Álvarez, F. J., \& López, A. M. (2013). Acción tutorial y orientación en el periodo de la transición de la Educación Secundaria a la Universidad. La orientación al alumnado de nuevo ingreso. Revista de Docencia Universitaria, 11(2), 221--241.

Eliasson, M., Berggren, H. y Bondestam, F. (2000) “Mentor programmes-a shortcut for women's academic careers?” en Higher Education in Europe, Vol. 25, núm. 2, pp. 173-179.

Hammersley, M. y Atkinson, P. (2009). Etnografía. Métodos de investigación. Barcelona: Paidós. 
Hayes, S. y Ljungberg, M. (2011). Dialogic exchanges and the negotiation of differences: female graduate students' experiences of obstacles related to academic mentoring. The qualitative report. Vol. 16 (3). pp. 682 - 710. Obtenido de http://nsuworks.nova.edu/tqr/vol16/iss3/4

García, E., Conejero, J. A., \& Díez, J. L. (2014). La entrada en la universidad: Un reto para la orientación académica. Revista de Docencia Universitaria, 12(2), 255--280.

Kagan, S. (1985). Dimensions of cooperative classroom structures, A: R. SLAVIN i cols.(eds), op. cit., p. 67-96

Lantarón, B. S. (2014). Los servicios de orientación profesional en las universidades españolas. Revista de Investigación en Educación, 12(1), 73--91

Lara Villanueva, R S; (2005). El aprendizaje cooperativo: un modelo de intervención para los programas de tutoría escolar en el nivel superior. Revista de la Educación Superior, XXXIV (1) 87-104. Obtenido de http://www.redalyc.org/articulo.oa?id=60411915008

Ministerio de Educación del Ecuador. (2012). Suplemento del Registro Oficial No. 955, de 3 de Marzo de 2017. Obtenido de http://educaciondecalidad.ec/ley-educacion-interculturalmenu/reglamento-loei-texto.html

Molina L; Rey C; Vall A; Clery A.; Santa María G (2016). La evaluación de las instituciones de Educación Superior. Revista Científica y Tecnológica UPSE, Vol III N.3 80.89. Recuperado de http://www.incyt.upse.edu.ec/revistas/index.php/rctu/article/view/201/pdf

Pérez Cusó, J; Martínez Juárez, M; (2015). Tutoria universitaria y servicios de orientacion: Dos realidades en un mismo contexto. Revista Electrónica Interuniversitaria de Formación del Profesorado, 18()$\quad 177-193 . \quad$ Obtenido de http://www.redalyc.org/articulo.oa?id=217036214013

Pérez-Montoro, Mario (2010) Arquitectura de la información en entornos web. Gijón: Trea, 2010. Obtenido de http://eprints.rclis.org/25021/1/Arquitectura $\% 20 \mathrm{de} \% 201 \mathrm{a} \% 20 \mathrm{informacion} \% 20 \mathrm{en} \% 20 \mathrm{ento}$ rnos\%20web.pdf

Pérez-Montoro, Mario (2004). Identificación del conocimiento organizacional: la propuesta epistemológica clásica [documento de proyecto en línea]. IN3: UOC. (Discussion Paper Series; DP04-001) Obtenido de http://www.uoc.edu/in3/dt/20390/index.html

Pérez Rojas, R. L. (2012). Universidad Veracruzana. Obtenido de http://www.uv.mx/dgdaie/files/2012/11/z-El-sistema-tutorial-en-la-UV.pdf

Ponce K, Torres E (2014). Diseño de un Sistema Web para la automatización de los procesos en la asignación de docentes tutores de aula en la Universidad Estatal de milagro periodo 2014. Obtenido de http://repositorio.unemi.edu.ec/xmlui/handle/123456789/1797 
Rey C., Balagué, N. y Falomir, V. (2006). Evaluación y gestión de la calidad de las bibliotecas universitarias. Marketing y comunicación: estado de la cuestión y propuestas de mejora. Madrid: EBIUN, 13 p

Sánchez García, M.F. y Guillamón Fernández, J.R.(Coords.) (2008). Situación actual de los servicios de orientación universitaria: estudio descriptivo. Revista de educación, 341, 329352.

Sanz Díaz, María Teresa (2012). Estudio de la acción tutorial como paso previo a la implantación del EEES en la Licenciatura de Administración y Dirección de Empresas de la Universidad de Huelva. Revista de Investigación Educativa, 30 (1), 145-160.

Villena D., Muñoz A., Polo. M. (2013). Revista de docencia universitaria. REDU: Revista de Docencia Universitaria, ISSN 1887-4592, No. 2, 2013. Red Estatal de Docencia Universitaria. Obtenido de https://dialnet.unirioja.es/servlet/articulo?codigo=4414095

Villena, Ma D., Muñoz, A. y Polo, T. (2013). La Unidad de Orientación de Centro como instrumento para la Orientación Universitaria. Revista de Docencia Universitaria. REDU. Número monográfico dedicado a Tutoría y sistemas de orientación y apoyo a los estudiantes, Vol.11 (2) Mayo-Agosto. pp. 43-62. Obtenido de http://www.red-u.net/ 